\title{
Vom Personal- zum Demografiemanagement? Der Einfluss der demografischen Frage auf das Personalmanagement
}

\author{
J. Reindl
}

\section{Abstract}

Der demografische Wandel in der Arbeitswelt war und ist vor allem ein Thema, das das Personalmanagement in den Unternehmen affiziert. Konzeptionell ist dadurch eine reiche Ernte eingefahren worden, die praktische Umsetzung hat hingegen gerade aufgrund der Präokkupation des Themas durch das Personalmanagement gelitten. Dennoch war die Befassung mit dem demografischen Wandel keine Spielwiese der ,Personaler', sie hat dazu beigetragen, das Personalmanagement aus seiner reaktiven Rolle herauszuführen und einen Paradigmenwechsel in der Personalpolitik von der Substanz- zur Potenzialbetrachtung des Personals einzuleiten.

\section{Einleitung}

Volker Volkholz hat den Anfang gemacht. Er hat als Erster die Frage, was der demografische Wandel für die Arbeitswelt bedeutet, aufgeworfen und die Belegschaften nicht wie üblich nach den Kriterien Qualifikation, Geschlecht, Beschäftigungsverhältnis etc. sortiert, sondern eine neue Kategorie ins Rampenlicht gestellt: das Alter (1). Zwar wurde schon seit Längerem über die älteren Arbeitnehmer"innen als Risikogruppe geforscht, aber die Alterskomposition eines Unternehmens war nicht der Rede wert, sie schien sich ja wie von selbst zu reproduzieren. Volkholz fielen bei seiner demografischen Betrachtung zwei beunruhigende Phänomene auf: geburtenschwache Jahrgänge, die die ausreichende Zufuhr des beruflichen Nachwuchses erschweren sowie eine gestauchte Altersstruktur in vielen Betrieben mit relativ wenig Jungen, sehr vielen Mittelalten und sehr wenig Alten, die auf einen unaufhaltsamen Alterungsprozess der Betriebe hindeutet. Er schlug Alarm und trat eine Lawine los: Forschungsprogramme wurden verkündet, wissenschaftliche Projekte in großer Anzahl aufgelegt, Konferenzen und Workshops in ähnlich großem Umfang veranstaltet, Be- 


\section{J. Reindl}

ratungsunternehmen und Stiftungen nahmen sich des Themas an, eine Publikation jagte die andere, neue Institutionen traten auf den Plan, die den Altersstrukturwandel und seine Folgen als exklusiven Beratungs- und Schulungsgegenstand bearbeiteten - kurzum, alle Welt hierzulande stellte sich die bange Frage, ob angesichts des dräuenden demografischen Wandels die Unternehmen so weitermachen könnten wie bisher oder ob neue Weichenstellungen erforderlich wären. Selten war wohl ein Agenda-Setting so erfolgreich, was seine Perzeption in der Öffentlichkeit angeht. Eine Zeit lang schien es so, als ob sich an dieser Frage die Zukunft entscheiden würde. Wie erfolgreich es allerdings in der Praxis und da in der Praxis der Unternehmen war, darüber wissen wir wenig, weil eine systematische Evaluation der Demografieaktivitäten noch aussteht bzw. nie in Betracht gezogen wurde. Wenn in diesem Text dennoch Aussagen dazu riskiert werden, dann sind es Aussagen, die auf der eigenen Mitwirkung an der Demografieagenda und auf der Durchführung etlicher diesbezüglicher Projekte in zahlreichen Unternehmen basieren (2). Es geht dabei nicht um den generellen Output und Outcome der vielen Initiativen und Vorhaben, sondern um die Effekte, die der Demografiediskurs auf das Personalmanagement als seiner wichtigsten Bastion in den Unternehmen hatte und hat.

\section{Demografie - eine Fundgrube für das Personalmanagement}

Die meisten Unternehmen, die über Projekte und externe Ansprachen mit der Demografiethematik konfrontiert wurden, haben zunächst zögerlich reagiert. Ihnen fehlte die Betroffenheit, ihre Altersstruktur war (noch) nicht kritisch und der Wille, in die Zukunft zu denken, war nicht sehr ausgeprägt. Eine Personengruppe im Unternehmen jedoch war wie elektrisiert von den Programmen, Konzepten, Projekten und Fragestellungen, die in die Betriebe hereinschwappten. Viele Personalverantwortliche haben sich die "Demografie“ richtig zu eigen gemacht, gleichsam als ob sie auf sie gewartet hätten. Sie haben offensichtlich in den durch den demografischen Wandel aufgeworfenen Problemstellungen zahlreiche Ansatzpunkte gefunden, ihre Vorstellung von Personalarbeit zu forcieren und womöglich im Unternehmen umzusetzen. Genau betrachtet ist ja das Personalmanagement, wenn es denn Macht und Gestaltungskraft hat, der ,natürliche' Ort, sich mit dem Altersstrukturwandel der Belegschaft und dem Alternsprozess im Unternehmen auseinanderzusetzen. Dort werden im Idealfall die Kriterien festgelegt, wer ins Unternehmen kommt, welchen Weg er/sie dort nimmt und wie er/sie aus dem Beruf heraustritt. 
Wir sind selbst Zeuge eines großen Experiments geworden, in dem Unternehmen des Maschinenbaus an das Demografiethema herangeführt wurden und dann selbstständig selbst gewählte und für wichtig befundene Fragestellungen bearbeitet haben (3). Es haben 47 Betriebe teilgenommen und hierbei fast ausschließlich ihre Personalverantwortlichen. Sie sind in einem ersten Workshop für das Thema sensibilisiert worden und sie haben aus dem Themenkranz ,Demografischer Wandel in der Arbeitswelt" ihr Projekt, das sie ein dreiviertel Jahr beschäftigten sollte, definiert. In einem zweiten Workshop nach getaner Arbeit haben sie Bericht erstattet über die Resultate, die sie erzielt hatten. Das Experiment gewährt einen spannenden Einblick in die Problemdefinitionen des Personalmanagements, in ihre Prioritätensetzung und in die Realisierbarkeit der entwickelten Konzepte.

Die teilnehmenden Betriebe sind auf drei Handlungsfeldern aktiv geworden: dem Berufseintritt, der Beschäftigung (Personalentwicklung und Gesundheit) und dem Berufsaustritt. Im Handlungsfeld ,Berufseintritt' haben sie auf die scheinbare Verknappung des Arbeitskräfteangebots reagiert und ihre Einstellungspolitik grundlegend hinterfragt. Scheinbar deshalb, weil der Rekrutierungsraum für die Firmen größer ist, als es auf den ersten Blick erscheint. Allzu oft suchen sie den passgenauen Bewerber, der jung, männlich, deutsch, gut qualifiziert ist und eine geradlinige Berufslaufbahn aufzuweisen hat. Weil um dieses Bewerberprofil aber längst ein ,war of talents' geführt wird, haben sich die Personalverantwortlichen an andere Arbeitsmarktgruppen erinnert: an Ältere, Frauen und praktisch begabte Jugendliche. Sie haben quasi einem Diversity-Ansatz in der Einstellungspolitik das Wort geredet und sie sind gut damit gefahren. Im Handlungsfeld ,Beschäftigung 'lag der Fokus auf Aktivitäten, die ein alternsgerechtes Arbeiten ermöglichen. Dazu zählen die Inangriffnahme von Laufbahn- bzw. Entwicklungswegeplanungen und Rotationsmodellen, die Forcierung der Weiterbildungsanstrengungen, die Einführung von Personalentwicklungsgesprächen, der Entwurf horizontaler Karrieren im Unternehmen, die Befassung mit den Perspektiven der Zielgruppe 50plus. Gerade auf diesem Handlungsfeld wurde zumindest konzeptionell eine reiche Ernte eingefahren. Da es für die meisten Teilnehmer*innen Neuland war, sprudelten hier die Ideen. Es war offensichtlich, dass die Personalverantwortlichen in puncto Gestaltung der Betriebslaufbahn einen großen Nachholbedarf sahen. Im Handlungsfeld ,Berufsaustritt' schließlich dominierten die Fragen des Wissenstransfers und Wissensmanagements. Sie hatten für viele teilnehmende Firmen eine hohe Priorität, weil aus ihrer Perspektive zahlreiche Mitarbeiterinnen und Mitarbeiter zu früh den Betrieb verlassen und wertvolles Know-how abziehen. Gerade in wissensintensiven Unterneh- 


\section{J. Reindl}

men, in denen die Arbeitskraft mit zunehmendem Alter ihren Wissenswert steigert, schmerzt dieser Aderlass besonders. Deshalb richtete sich ein Hauptaugenmerk auf die Aufbewahrung und Weitergabe dieses Wissens, wobei die anfängliche Euphorie der Teilnehmer*innen für Wissensmanagementsysteme mehr und mehr dem Ansatz des interpersonalen Wissenstransfers über die Bildung von Tandems, Patensysteme und Nachfolgeplanungen gewichen ist.

Als Moderatoren und Begleiter dieses Experiments waren wir überrascht, mit welcher Energie, welchem Ideenreichtum und welcher Kreativität die Personalverantwortlichen das Demografiethema angepackt haben. Für sie tat sich damit offensichtlich eine Bühne auf, auf der sie aus dem Schatten, in dem sie häufig agieren, heraustreten und in die Gestaltungsrolle wechseln können. Das Personalmanagement muss ja zum einen oft exekutieren, was andernorts im Unternehmen entschieden wurde, es hat wenig Einfluss auf die Ausarbeitung der Unternehmensstrategie (4). Zum anderen beackert es gewöhnlich vor allem die Felder des Berufseinund -austritts sowie die Verwaltung des Personals mit einiger Professionalität und Routine, aber die Personalentwicklung und die Gesamtentwicklung des Belegschaftskörpers bleiben häufig Stückwerk oder fallen aufgrund des Aktualitätsdrucks unter den Tisch (5). Die Demografieinitiative hat den Verantwortlichen die Chance eröffnet, neue Akzente in der Personalpolitik weg von der Administration und hin zur Entfaltung des Personals zu setzen, den eigenen Status aufzuwerten und als Personalmanagement Anerkennung bei den anderen Managementdisziplinen zu erhalten.

\section{Die Grenzen des Demografiemanagements}

Den Personalverantwortlichen, die an der Demografieinitiative teilgenommen haben, ist etwas Ähnliches widerfahren, was in vielen Demografieprojekten zu besichtigen war. Sie haben konzeptionell hoch originell gearbeitet, sie waren in ihrem Element, weil sie endlich auf der Klaviatur des gesamten Personalmanagements spielen konnten, sie haben auch - weil viele Mittelständler dabei waren - in der dort typischen Manier schnell Dinge auf den Weg gebracht wie etwa die Revision bisheriger Stellenausschreibungen oder die ergonomische Verbesserung bestimmter Arbeitsplätze, aber der große Wurf, das Unternehmen demografiefest zu machen, ist ausgeblieben. Vieles, was sie sich vorgenommen haben, ist auf dem geduldigen Papier geblieben, einiges ist im Alltagsstress des Tagesgeschäfts versandet. Zwei Gründe zeichnen sich dafür verantwortlich, dass ihr Demogra- 
fievorhaben auf halbem Weg stecken geblieben ist: ein strategischer und ein struktureller.

Die Demografieinitiative war eine Initiative des Personalmanagements und entsprechend haben die ,Personalerinnen' und ,Personaler' die Themen gesetzt, die ihre Themen waren: also Personalgewinnung, Personalentwicklung, Qualifizierung, Nachfolgeplanung, Wissenstransfer. Gemeinhin gelten sie als die weichen Issues, die sich schlecht rechnen lassen und die so lange entfaltet werden können, so lange sie nicht die harten Issues das Leistungsregime, die Produktivität, die Kosten-, Termin- und Rentabilitätsziele - tangieren. Über diese aber wacht das operative Management des Wertschöpfungsprozesses, das aufgrund der Dezentralisierung der Unternehmen (Profit-Center, Divisionalisierung etc.) mächtiger geworden ist und sich ungern von außen dreinreden lässt. (6) Wenn von HR etwa Rotationsmodelle vorgeschlagen werden, schlägt ihnen sofort das Argument entgegen, dies gehe mit Produktivitätsverlusten einher, weil auf dem neuen Arbeitsplatz Einarbeitungskosten entstünden. Wenn mehr Qualifizierung und Schulung angemahnt wird, verweist das operative Management auf die fehlende Zeit, die dringend für die Erfüllung der Ziele gebraucht würde. Wenn in den FuE-Bereichen neue Karrierewege (sog. Fachkarrieren) erwogen werden, steht die Befürchtung im Raum, dies würde das Budget sprengen, weil dadurch auch die Gehälter steigen könnten. Es ist bezeichnend, dass in den allermeisten Demografievorhaben die Leistungspolitik eine Leerstelle geblieben ist und das operative Management nicht oder nur sehr unzulänglich in die Pläne des Personalmanagements einbezogen worden ist. Die Konzepte sind quasi im ,luftleeren Raum' entwickelt worden unter Absehung der Kalküle und Überlegungen des operativen Managements. U.E. war dies ein strategischer Fehler, der mitverantwortlich ist für das Scheitern ambitionierter Vorhaben. Eine Lehre daraus ist, dass Veränderungen im Personaleinsatz, in den Karrierewegen und im Arbeitszuschnitt, die ein gesundes und produktives Altern im Unternehmen befördern, nur im engen Schulterschluss mit den Verantwortlichen des Wertschöpfungsprozesses machbar sind. Sie müssen überzeugt werden, dass ein anderes Arbeiten kein unproduktiveres ist und dass es ihnen nützt, wenn sie Beschäftigte haben, denen die Möglichkeit eröffnet wird, ihr Potenzial über einen engen Aufgabenzuschnitt hinaus zu erweitern (7).

Der zweite Grund, warum viele Demografieprojekte festgefahren sind oder kaum Wirkung erzielt haben, ist ein struktureller. Die Alters- und Alternsfrage ist - anders als etwa im Öffentlichen Dienst oder in nicht profitgetriebenen Organisationen - im kapitalistischen Unternehmen eine abgeleitete Frage. Im Zentrum aller Überlegungen steht, auf welche Weise Unternehmen durch die Herstellung von Gütern oder Dienstleistungen Ge- 


\section{J. Reindl}

winn machen können. Im Zentrum steht nicht, wie ein optimaler Belegschaftsaufbau aussehen könnte und auch nicht, wie die Belegschaft gesund altern kann. Diese Fragen bekommen erst eine Relevanz, wenn der Zweck des Unternehmens gefährdet ist, weil es nicht die benötigten Fachkräfte bekommt, weil die guten Leute nicht gehalten werden können, weil bald Wissensträger en gros in den Ruhestand gehen oder weil die Krankheitsquote zu hoch ist. Treten solche Konstellationen auf, dann besteht eine gute Chance, das Demografiethema im Unternehmen zu verankern. Fehlen sie hingegen, dann ist das Auflegen einer Demografieagenda ein Kampf mit Windmühlen. Die dem Demografiemanagement immanente Vorstellung, man könne das Thema autonom als solches fahren und damit präventiv gegen zukünftige Verwerfungen handeln, geht in die Irre. Der erfolgversprechendere Weg besteht darin, es mit den Fragestellungen, Problemen und Zukunftsthemen zusammenzuschalten, die das Unternehmen umtreiben und in Atem halten, sei es aktuell die Digitalisierung oder seien es Umstrukturierungen/Reorganisationen, die Einführung neuer Steuerungsprinzipien (indirekte Steuerung), der Aufbau von Team- und Gruppenarbeit etc. (8). Wenn ein Personalmanagement sich in solche Veränderungsprozesse hineinbegibt und zu bedenken gibt, was diese mit älteren Mitarbeiterinnen und Mitarbeitern machen und was daran alterns- und altersgerecht ist oder was nicht, dann hat es ungleich mehr Chancen, mit seinen Ideen, ein gesundes und produktives Altern zu ermöglichen, durchzudringen, als wenn es eine abstrakte Agenda gegen die zukünftigen Risiken des demografischen Wandels aufstellt.

\section{Das demografische Projekt als Professionalisierungsschub des Personalmanagements und als Modernisierungsschub der Personalpolitik}

Auch wenn diese Befunde zu den Wirkungen der Demografieaktivitäten ernüchternd klingen, so wäre es dennoch grundverkehrt, von ihrem Scheitern zu reden. Mehr als kurzfristige Erfolge, die bei diesem Langfrist-Thema ohnehin nicht leicht zu erwarten sind, wiegen die indirekten Effekte, die von ihnen ausgingen. Die Befassung mit dem demografischen Wandel und die Begegnung mit den im Rahmen der bundesweiten Aktivitäten entwickelten Konzepten und Werkzeugen haben zu einer Stärkung des Personalmanagements und zu einer Neuausrichtung der Personalpolitik geführt. Sie waren Vehikel und Katalysatoren eines Modernisierungsprozesses, Geburtshelfer einer modernen Personalpolitik.

Die Personalpolitik, wie sie in vielen Unternehmen verbreitet war, war eine weitgehend defensive, wenn sie sich nicht gar auf reine Personalver- 
waltung beschränkt hat. Sie hat die technisch-ökonomische Strategie des Unternehmens nachvollzogen und ihre möglichen negativen Wirkungen abgefedert. Sie hat im Falle des Personalbedarfs den Arbeitnehmertypus eingestellt, den man schon immer gehabt hat, sie hat im Falle eines Ungleichgewichts zwischen vorhandenen und benötigten Qualifikationen Anpassqualifizierung betrieben, sie hat vertikale Karrieren entworfen, über deren Einnahme der Meister, die Bewährung in der Arbeit und das Alter entschieden haben, sie hat den Führungskräftenachwuchs geplant und sie hat schließlich nach Wegen gesucht, die ältere Mitarbeiterin oder den älteren Mitarbeiter vorzeitig oder gleitend in den Ruhestand zu verabschieden. Dass es damit nicht sein Bewenden haben kann, ist spätestens dann klargeworden, als im Zuge moderner Managementkonzepte die Ressource Mensch ins Zentrum rückte und der Arbeitnehmer*innen als ,Unternehmer im Unternehmen' angerufen wurde (9). Den Beschäftigten wurde jetzt wesentlich mehr abgefordert als in der alten Welt, in der sie vorbestimmte Aufgaben abzuarbeiten hatten, sie sollten autonomer, freier, selbstverantwortlicher, flexibler werden und an ihren Arbeitsplätzen oder in ihren Teams so handeln, als ob ihnen selbst das Unternehmen gehören würde. Dieser neue Arbeitshabitus sollte bei den Beschäftigten vor allem durch neue Organisations- und Steuerungsmethoden erzeugt werden, die sie näher an den Kunden und den Markt heranrücken oder die den Markt im Unternehmen durch Kennzahlen, Benchmarks, Kunden-LieferantenBeziehungen simulieren. Der Part des Personalmanagements war es, die Beschäftigten durch entsprechende Qualifizierung dafür fit zu machen und sie von den Vorteilen der neuen Methoden zu überzeugen. Auf der Suche nach ihrer Rolle in diesem Prozess der Modernisierung der betrieblichen Herrschaft kam ihnen das im Demografiediskurs angehäufte Konzept- und Handlungswissen entgegen, wenngleich es einen anderen Ausgangspunkt hatte. Es befasst sich ja im Kern mit der Frage, wie die Arbeitsfähigkeit und Produktivität der Mitarbeiter*innen über ein Arbeitsleben lang gefördert werden kann und wie diese eigenständig, aber unterstützt vom Unternehmen, einen Berufsweg finden können, der ihr Potenzial zur Entfaltung kommen lässt, anstatt es vorzeitig durch einseitige und immer gleiche Arbeit zu ersticken. Funktionieren kann eine solche arbeitsbiographische Perspektive nur, wenn zwischen Mitarbeiter*innen und Personalverantwortlichen viel darüber kommuniziert wird.

Der demografische Wandel hat so gesehen stark mit dazu beigetragen, einen Paradigmenwechsel in der Personalpolitik einzuleiten. Es zieht mit ihm und mit der Aufwertung des „Faktor Mensch“ eine neue Betrachtungsweise des Personals in die Unternehmen ein, die Folgen für ihre Behandlung und den Umgang mit ihnen hat. In ihr wird das Personal nicht 


\section{J. Reindl}

mehr als eine statische, sondern als eine dynamische Größe perzipiert. Die Belegschaft ist nicht Substanz, sondern Potenzial. Die Substanzbetrachtung geht von gegebenen Anforderungs- und Qualifikationsstrukturen aus, von einer grundsätzlichen Passung zwischen betrieblicher Qualifikationsnachfrage und betrieblichem Qualifikationsangebot, die allenfalls kurzfristiger Korrekturen bedarf. Hierfür werden dann Anpassungs- oder Ad-hoc-Qualifizierungen in Form von Schulungen und Trainings durchgeführt. Sie geht weiter davon aus, dass die Substanz jederzeit wieder aufgefüllt werden kann: durch Eigenausbildung oder durch Beschaffung der benötigten Qualifikationen vom Arbeitsmarkt. Die Potenzialbetrachtung hingegen dynamisiert die Anforderungs- und Qualifikationsstrukturen. Sie nimmt die zukünftigen Anforderungen (SOLL) in den Blick und gleicht sie mit der gegenwärtigen Qualifikationsstruktur (IST) ab. Die Lücke, die dabei zwischen SOLL und IST klafft, veranlasst das Personalmanagement allerdings nicht, alle Rekrutierungshebel in Bewegung zu setzen und sich frühzeitig mit den benötigten Qualifikationen einzudecken. Es wirft vielmehr einen zweiten vertieften Blick auf das IST und stellt fest, dass im Prinzip im gegenwärtigen Belegschaftskörper das Potenzial da ist, den zukünftigen Anforderungen gerecht zu werden. Der zweite Blick ruht nicht mehr bloß auf den formellen Qualifikationen, sondern er richtet die Aufmerksamkeit auf den Arbeitseinsatz und die latent bleibenden, weil nicht abgeforderten Fähigkeiten und Fertigkeiten. Er heftet sich an die Kompetenz der Mitarbeiter*innen, an ihr Know-how, ihre Bewältigung von Anforderungen, ihr Erfahrungswissen, ihre Qualifikationskarriere, ihre verschütteten Möglichkeiten, kurzum ihr Potenzial, das allemal größer ist als der im Arbeitsprozess abgeforderte Kompetenzausschnitt. In der Potenzialbetrachtung wird deshalb so intensiv auf den gegenwärtigen Qualifikationskörper geschaut, weil ihre Vertreter*innen von einem wachsenden Mismatch zwischen zukünftig benötigten und gegenwärtig fabrizierten Qualifikationen ausgehen und weil sie sich die Knappheitshypothese schon zu eigen gemacht haben. Wenn die Unternehmen die Qualifikationen nicht mehr so ohne Weiteres auf dem Arbeitsmarkt kaufen können, dann müssen sie aus dem bestehenden betrieblichen Qualifikationsstock schöpfen und diesen fortentwickeln. Sie müssen beginnen, anstatt situativ und temporär, in den Dimensionen von Arbeitsbiographien und von langfristigen Entwicklungswegen der Mitarbeiterinnen und Mitarbeiter zu denken. Sie dürfen die Arbeitskraft nicht mehr kurz- und mittelfristig verschleißen, sondern sie müssen sie auf lange Sicht fördern und entwickeln.

Der Potenzialansatz umfasst idealtypisch vier Stufen. Auf jeder warten auf die Personalverantwortlichen neue Aufgaben und Herausforderungen, die nur mehr wenig zu tun haben mit der klassischen Personalarbeit. 
- Die erste Stufe besteht in einer IST-Aufnabme zur gegenwärtigen Performance der Humanressourcen. Sie beinhaltet eine Erhebung der Qualifikationsstruktur, der Tätigkeitsstruktur sowie der Kompetenzstruktur. Vergleichsweise einfach lässt sich noch die Qualifikationsstruktur erfassen (formelle Berufsabschlüsse und Weiterbildungen). Schwieriger wird es schon bei der Tätigkeitsstruktur (i.e. die gegenwärtige Anforderungsstruktur), weil i.d.R. Stellenbeschreibung und tatsächlicher Arbeitsinhalt nicht zusammenfallen. Die Anamnese verkompliziert sich noch einmal, wenn die Kompetenz der Mitarbeiterin oder des Mitarbeiters ins Spiel kommt, also die Art und Weise, wie er seine Arbeit meistert. Kompetenz ist immer auch etwas Zugeschriebenes, eine Bewertung und von daher nicht aus der objektiven Abbildung der Belegschaft in der Beschäftigtenstatistik ablesbar. Sie lässt sich nur erfassen, wenn die üblichen quantitativen Verfahren durch qualitative, also kommunikative Verfahren ergänzt werden.

- Der nächste Schritt ist die Bedarfsanalyse. Sie ist in die Zukunft gerichtet, liest aus gegenwärtigen Trends zukünftige Entwicklungen heraus und formuliert den zu erwartenden betrieblichen Bedarf an Qualifikationen und Kompetenzen. Bedarfsanalysen sind notwendig mit Unsicherheit behaftet, da sie eine Prognose der technologischen Entwicklung und der künftigen Gestalt der Arbeit sowie der daraus resultierenden zukünftigen Anforderungsprofile anstellen müssen. Sie sind nur sinnvoll zu erstellen im Abgleich mit den unternehmenspolitischen Strategien und Plänen. Für das Personalmanagement eröffnet sich damit die Chance, eine gestaltende Rolle bei der personellen und sozialen Auslegung der unternehmerischen Strategie- und Geschäftsprozesse zu spielen, aus der Reactio herauszukommen.

- Auf der dritten Stufe, der Potenzialanalyse, wird danach gefragt, wie mit den gegenwärtigen Ressourcen die Herausforderungen der Zukunft bewältigt werden können und was zu unternehmen ist, um das Personal dafür vorzubereiten. Sie hat eine subjektive und eine objektive Dimension. Die Verantwortlichen nehmen zum einen eine Einschätzung der Entwicklungsmöglichkeiten ihrer Mitarbeiter*innen, d.h. vor allem ihres latenten Potenzials, vor. Zum andern fragen sie nach der Lernhaltigkeit und Lernförderlichkeit der gegenwärtigen Arbeitsplätze und Arbeitsarrangements und sie entwerfen langfristige Entwicklungs- und Qualifizierungsmuster, die ihren Ankerpunkt in der Arbeitsbiographie der Beschäftigten haben. In Rede stehen bei einer solchen Entwicklungswegeplanung Karrierewege (vertikale Karrieren), aber auch, weil die Aufstiegswege zusehends verstopft sind und die Führungspositionen aufgrund der Abflachung der Hierarchien weniger werden, hori- 
zontale Laufbahnen und Fachkarrieren. Die Potenzialanalyse gibt es schon in den Unternehmen in Form der Führungskräftenachwuchsplanung. Sie ist aber als eine Methode, die sich tendenziell auf alle Beschäftigten bezieht, Neuland.

- Das Mitarbeitergespräch als vierte Stufe ist die entscheidende Station in diesem neuen personalpolitischen Ansatz. Dort gleichen das Management und die Mitarbeiterin oder der Mitarbeiter ihre Ziele ab, vereinbaren die Wege dorthin, die Zwischenstopps und die Maßnahmen, die ergriffen werden müssen, um die Zielerreichung abzusichern. Mitarbeitergespräche im hier verstandenen Sinne unterscheiden sich erheblich von den bekannten Kommunikationsformen wie etwa Leistungsbeurteilungsgespräche oder Vorladungen ins Personalbüro. Betrieb und Mitarbeiterin bzw. Mitarbeiter treten im Mitarbeitergespräch, das auch Qualifizierungs-, Förder-, Personalentwicklungs- oder Zukunftsgespräch heißen kann, gewissermaßen in eine neue Beziehung, sie handeln quasi ihre gemeinsame Zukunft aus. Funktionieren kann das nur, wenn sie auf gleicher Augenhöhe kommunizieren, wenn im Dialog die Asymmetrie zwischen Arbeitgeber*innen und Arbeitnehmer*innen aufgehoben ist, ein ,herrschaftsfreier Diskurs" versucht wird.

- Die Maßnahmen schließlich, die als Resultat der Analysen und Gespräche festgelegt werden, haben - nimmt man den arbeitsbiographischen Ansatz ernst - einen anderen Zuschnitt als die üblichen Ad-hoc-Schulungen. Es geht beim ,lebenslangen Lernen' um die Entwicklung eines individuellen Qualifizierungs-Curriculums, dessen Elemente das Lernen im Arbeitsprozess durch lernförderliche Arbeits(biographie)-Gestaltung, eine Qualifizierungskette von aufeinander abgestimmten Qualifizierungsbausteinen sowie der Wissens- und Erfahrungstransfer zwischen Positionsinhaber"in und Nachfolger"in sind.

Eine nachhaltige Personalpolitik, wie sie hier skizziert worden ist, ist gewiss noch nicht die Regel in den Unternehmen. Paradigmen, die ja im Wesentlichen Kulturen sind, verändern sich nur langsam. Aber die Vorstellung, dass Personalmanagement mehr sein kann als die bürokratische Verwaltung der ohnehin geschehenden Personalbewegungen im Unternehmen, dass sie die gezielte Formung des Belegschaftskörpers und die Entfaltung des Humanpotenzials zum Inhalt haben könnte, ist in der Welt und orientiert die Personalverantwortlichen zusehends. Der Demografiediskurs hat diese Entwicklung kräftig angeschoben, weil er durch seine Szenarien der Verknappung des Nachwuchses und der Alterung der Belegschaften das Erfordernis für die Betriebe herausgearbeitet hat, mehr aus 
dem Eigenen zu schöpfen und die Human-Ressourcen pfleglich zu behandeln.

\section{Fazit: Was bleibt?}

Wer sich von der Demografieoffensive in der Arbeitswelt bahnbrechende Innovationen in der Arbeits- und Personalpolitik erwartet hat, der muss erfreut und enttäuscht zugleich sein. Erfreut, weil wirklich ein neuer Denkansatz zum Tragen gekommen ist und auf seiner Grundlage viele innovative Konzepte und Tools entwickelt wurden (10). Enttäuscht, weil das Handlungsprogramm des gesunden und produktiven Alterns nur selten wirklich Gestalt angenommen hat und oft Stückwerk oder Insellösung blieb. Die Frustration liegt allerdings eher aufseiten der überbetrieblichen Promotoren der Demografieagenda und der damit befassten Wissenschaft, weniger aufseiten der betrieblichen Praktiker, die um die Schwierigkeiten wissen, den sog. ,weichen Themen' zum Durchbruch zu verhelfen. Es ist ein Bohren dicker Bretter, immer wieder mit Rückschlägen verbunden, aber der neue Denkansatz hat sich in den Köpfen vieler betrieblicher Akteure festgesetzt und er beeinflusst ihr Handeln.

Darin, in der Attraktion, die das Gedankengut der alternsgerechten Arbeit v.a. auf das Personalmanagement ausgeübt und dadurch ihren Aktionsradius erweitert und ihre Personalpolitik befruchtet hat, liegt der wohl größte Nutzen der Demografieaktivitäten. Wenn wir die klassischen personalpolitischen Handlungsfelder durchdeklinieren, ist es in vielen Unternehmen, vor allem denen, die sich an der Demografieoffensive beteiligt haben, zu Neuausrichtungen, Veränderungen, Ergänzungen gekommen. Und auch da, wo wenig aktive Berührung mit den Demografieaktivitäten war, ist zumindest durchgedrungen, dass es da eine Programmatik gibt, die abweicht vom Mainstream des Personalmanagements.

Wenn wir die Bausteine einer alter(n)sgerechten Personalpolitik, die Fuß in vielen Betrieben gefasst haben, benennen sollten, dann kristallisieren sich die folgenden Module heraus:

(1) Demografisches Monitoring: Darunter ist die permanente Beobachtung der betrieblichen Altersstrukturentwicklung zu verstehen und ihr Abgleich mit der Personalstatistik sowie der betrieblichen Bedarfsanalyse. Das demografische Monitoring ist ein Frühwarnsystem, das drohende demografische Verwerfungen rechtzeitig meldet.

(2) Diversity Management in der Rekrutierungspolitik: Darunter wird die Selbstreflexion der betrieblichen Einstellungskriterien gefasst sowie 
die Öffnung der innerbetrieblichen Arbeitsmärkte für Ältere, Frauen, Migranten und andere bislang vernachlässigte Arbeitsmarktgruppen.

(3) Das Mitarbeitergespräch als Grundlage der Personalentwicklung: Damit wird auf das Erfordernis fokussiert, Mitarbeiterinnen und Mitarbeiter zu binden, indem ihnen Entwicklungsmöglichkeiten geboten werden. Das Mitarbeitergespräch ist das geeignete Forum, um die beruflichen Interessen der Mitarbeiterin bzw. des Mitarbeiters und die Verwertungsinteressen des Betriebes abzustimmen und auszubalancieren.

(4) Eine Arbeitseinsatzpolitik des Wechsels: Damit wird die Bedeutung von Laufbahnen und Entwicklungswegen ebenso unterstrichen wie die des Abwechslungsreichtums in der Arbeit. Der Wechsel ist gut für die Gesundheit, für die persönliche Weiterentwicklung, für das ,lebenslange Lernen' und für den Wissenstransfer

(5) Eine Wissenspolitik des Transfers: Darunter sind alle Formen zu begreifen, in denen Wissen nicht das Privateigentum der Arbeitsperson bleibt, sondern ,fließt und, wandert $\mathrm{t}^{6}$. Wissen wird durch Austausch und Begegnung wertvoller und die Kreuzung unterschiedlicher Wissenskulturen (Jung und Alt) begründet ein Milieu, in dem Innovationen nicht nur reifen, sondern auch in gute Produkte münden.

(6) Ein intergenerationelles Personalmanagement: Damit ist die vernünftige Kombination der Generationen im Betrieb gemeint. Zum einen geht es um eine einigermaßen ausbalancierte heterogene Altersstruktur, zum anderen um das In-Beziehung-Bringen der Generationen (z.B. in der Einarbeitung oder Nachfolgeplanung).

(7) Die ,Humanisierung' des Berufsaustritts: Darunter ist eine bewusste Gestaltung der Ausgänge aus dem Betrieb zu verstehen. In Rede steht ein einvernehmlicher Abschied und ein ,Weiterleben' der Mitarbeiterin bzw. des Mitarbeiters im Unternehmen nach seinem Abschied: in Form des Wissens, das er zurückgelassen hat, in Form punktueller Engagements, in Form gezielter Beziehungspflege.

(8) Symbolpolitik: Dies meint die Energie freisetzende Wirkung, die eine wertschätzende Behandlung älterer Mitarbeiterinnen und Mitarbeiter auf die übrige Belegschaft hat. Sie deutet ihre Zukunft im Lichte des heutigen Umgangs mit älteren Beschäftigten. Sie antizipiert, was sie später zu erwarten hat und richtet sich in ihrem Verhalten darauf ein.

Diese Module sind die Frucht der vielen Aktivitäten zum „Demografischen Wandel in der Arbeitswelt“. Sie haben sich von ihrem Ursprung inzwischen abgelöst und Eingang gefunden in das Spektrum personalpolitischen Handelns. Dies heißt selbstredend nicht, dass sie überall das personalpolitische Handeln prägen, aber das Personalmanagement kann nicht 
mehr hinter die in den Modulen zum Ausdruck kommenden Einsichten zum Zusammenhang von Arbeit und Altern zurückgehen. Es kennt sie. $\mathrm{Ob}$ es danach handelt oder handeln kann, steht auf einem anderen Blatt.

\section{Anmerkungen}

(1) Volkholz hat schon früh immer wieder auf die kommenden demografischen Verwerfungen hingewiesen. Es waren "graue Papiere“, die eine enorme Wirkung auf die Projektträger und das Forschungsministerium hatten. Vgl. Volkholz (1991) und Volkholz (1997).

(2) Die wichtigsten Vorhaben, an denen der Verf. beteiligt war, waren das Verbundprojekt „Innovation, Belegschaftsstrukturen und Altern im Betrieb“, das in 35 Unternehmen Fallstudien durchgeführt hat (Köchling u.a. 2000), das Projekt „Ältere in Innovationsprozessen“, an dem 11 Betriebe beteiligt waren (Grewer u.a. 2007), die „Demografie-Initiative des VDMA“ mit 47 teilnehmenden Betrieben (Reindl u.a. 2004) sowie das Verbundprojekt LEGESA (Lebenslang gesund arbeiten) mit fünf geförderten Praxispartnern (Reindl 2012).

(3) Der offizielle Titel der Demografieinitiative des VDMA lautete: „Betriebliche Strategien für eine alternsgerechte und generationenübergreifende Arbeits- und Personalpolitik im Maschinen- und Anlagenbau."

(4) Zum Status und zur Funktionsweise des Personalmanagements vgl. Schreyögg 1987.

(5) $\mathrm{Zu}$ den Professionalisierungsprozessen im Personalmanagement und zur Absorbierung des Personalmanagements durch das Tagesgeschäft vgl. Wächter 1987 und Gaugler 1995.

(6) Eine Übersicht über den Wandel der Unternehmensorganisation und über die Veränderungen, die sich für die verschiedenen Managementdisziplinen ergeben haben, findet sich bei Sperling 1997.

(7) Bei der Firma SICK, die an etlichen Demografieprojekten beteiligt war, lässt sich dieser Zusammenhang exemplarisch an zwei Themenfeldern studieren. Während im Themenfeld "Schichtarbeit" schnell Erfolge eingefahren wurden und die Nachtschicht für Ältere abgeschafft wurde, hakte es im Themenfeld „Fachkarrieren“ erheblich. Der Grund war, dass im Falle der Schichtarbeit das operative Management von Anfang an eingebunden war, während im Falle der Fachkarriere den Verantwortlichen von oben ein Konzept präsentiert wurde, das sofort die Widerstandsgeister wachrief. Vgl. Reindl 2012, S. 75-116. 
(8) Ähnlich argumentieren Anlauft und Feldes in diesem Band bei ihrer Bilanzierung der tariflichen Befassung mit dem demografischen Wandel.

(9) Vgl. dazu Pongratz und Voß 2003 sowie Peters und Sauer 2005.

(10) Beispielhaft seien hier die Bundesanstalt für Arbeitsschutz und Arbeitsmedizin (BAuA), die Initiative Neue Qualität der Arbeit (INQA) sowie das Projekt „Psychische Gesundheit in der Arbeitswelt“ (psyGa) genannt, die zahlreiche Ausarbeitungen, Broschüren und Handreichungen zu den Fragen des demografischen Wandels in der Arbeitswelt erstellt haben.

\section{Literatur}

Gaugler, E. (1995): Personalmanagement in schwierigen Zeiten. In: C. Scholz, H. Oberschulte (Hrsg.), Personalmanagement in Abhängigkeit von der Konjunktur. München und Mering S. 1-14.

Grewer, G., Matthäi, I., Reindl, J. (2007): Der innovative Ältere. Warum die Entwickleruhr länger als sieben Jahre tickt. München und Mering.

Köchling, A., Astor, M., Fröhner, K.-D., Hartmann, E., Hitzblech, T., Jasper, G., Reindl, J. (2000): Innovation und Leistung mit älterwerdenden Belegschaften. München und Mering.

Peters, K., Sauer, D. (2005): Indirekte Steuerung - eine neue Herrschaftsform. Zur revolutionären Qualität des gegenwärtigen Umbruchprozesses. In: H. Wagner (Hrsg.), Rentier ich mich noch? Neue Steuerungsformen im Betrieb. Hamburg, S. 23-58.

Pongratz, H., Voß, G. (2003): Arbeitskraftunternehmer. Erwerbsorientierungen in entgrenzten Arbeitsformen. Berlin.

Reindl, J. (2000): Betriebliche Innovationsmilieus und das Alter(n) der technischen Intelligenz. In: A. Köchling u.a. (Hrsg.), Innovation und Leistung mit älterwerdenden Belegschaften. München und Mering, S. 265-316.

Reindl, J., Feller, C., Morschhäuser, M., Huber, A. (2004): Für immer jung? Wie Unternehmen des Maschinenbaus dem demografischen Wandel begegnen. Frankfurt.

Reindl, J. (2012): Paradoxe Freiheit, gestörter Sinn. Verstehende Prävention in der modernen Arbeitswelt. Berlin.

Schreyögg, G. (1987): Verschlüsselte Botschaften. Neue Perspektiven einer strategischen Personalführung. In: Zeitschrift Führung und Organisation, Nr.3, S. $151-158$.

Wächter, H. (1987): Professionalisierung im Personalbereich. In: Die Betriebswirtschaft, Jg. 47 (2), S. 141-150. 
Volkholz, V. (1991): Alter, Leistung, Gesundheit. Eine Anregung zur Diskussion über die Tarifreform 2000. In: Graue Reihe der Hans Böckler Stiftung (HBS), Nr. 35. Düsseldorf.

Volkholz, V. (1997): Betriebliche Folgen einer verlängerten Erwerbstätigkeitsdauer oder: Was bei den Konsolidierungsversuchen der Rentenfinanzierung (bislang) nicht diskutiert worden ist. In: G. Jasper, V. Volkholz, Änderung der betrieblichen Alterszusammensetzung: Gestaltungsherausforderungen. Graue Reihe der HBS, Nr. 132. Düsseldorf. 
